\title{
Deux acariens parasites de Gallus domesticus Lin. à Madagascar (Bdellonyssus bursa Berlese, 1888, et Tyroglyphus sp.)
}

\author{
par S. GRÉTILLAT
}

Dans une précédente note, nous avons signalé l'existence d'un Bdellonyssus Da Fonseca à Madagascar, trouvé sur des poules d'un élevage avicole de la région de Tananarive.

Depuis lors, les travaux de détermination ont montré qu'il s'agit de Bdellonyssus bursa Berlese, 1888, de la famille des Macronyssidae Oudemans, 1936.

Cet acarien mésostigmate est très répandu en Amérique du Sud, où il a été déçouvert pour la première fois sur des volailles par Balzan, en 1888 , et décrit la même année par Berlese sous le nom de Leiognathus bursa. En 1915, Hirst signale que ce parasite est très largement répandu dans les régions tropicales et sublropicales, et lui donne le nom de Leiognathus morsitans. En 1923, Ewing le décrit et l'appelle Liponyssus bursa Berlese, 1888; il donne une bonne description du mâle et de la femelle.

Da Fonseca en 1947, dans une excellente monographie de la famille des Macronyssidae, l'appelle Bdellonyssus bursa.

Bdellonyssus bursa ou « tropical mile » des auteurs anglo-saxons est très répandu en Amérique du Nord et du Sud ainsi qu'en Afrique du Sud où il parasite les volailles et 'même parfois l'homme. Hirst (1915) fait remarquer que cet acarien est beaucoup plus répandu qu'on ne le croit et qu'il est souvent confondu avec Dermanyssus gallinae De Geer, 1776.

Il a été trouvé en Gambie (Docteur J.-J. Simpson, 1011) sur des poules, en Nigeria (J.-M. Dalziel, 1912) sur des poulets atteints de spirochétose aviaire. aux Comores (Mayotte) sur un hibou (C.-F. Leigh). A Maurice, il est très commun sur les poules ainsi que sur les moineaux (Passer domesticus). A ce sujet, Ewing, en 1911, met l'accent sur le rôle que peuvent jouer les moineaux dans la dissémination des acariens hématophages des volailles, et en particulier d'un acarien qu'il appelle Dermanyssus avium. Hirst (1915) doute qu'il y ait identité entre ce parasite et $B$. bursa. Il pense qu'il s'agit d'une espèce différente.

Au Nyassaland, le Docteur J.-E.-S. Old a observé de nombreux spécimens de $B$. bursa dans des nids de poules.

Au Zoulouland, M. W.-M. Jones l'a trouvé parasitant massivement les volailles.

A Madagascar, il n'avait pas encore été signalé. Il est cependant très répandu dans les élevages avicoles malgaches où il cause d'importants dégâts surtout parmi les jeunes poulets. Il peut occasionnellement, comme en Amérique du sud et à Zanzibar (Docteur W.-M. Aders), parasiter l'homme. En décembre 1955, à Tananarive, une maison a été envahie par $B$. bursa qui a attaqué les occupants et en particulier un bébé. Après détermination du parasite en cause, l'enquête sur place a montré que les acariens tombaient du plafond d'une pièce au-dessus de laquelle avaient été parquées des poules un an auparavant. $B$. bursa attaquerait l'homme lorsqu'il devient trop nombreux sur les volailles ou lorsqu'il a été soumis à un jeûne prolongé, ce qui semble s'être produit'à Tananarive.

Chez l'homme, les premiers symptômes observés lors de l'invasion des parasites sont une forte irritation de l'épiderme accompagnée d'un prurit plus ou moins intense; puis apparaissent des éruptions cutanées avec de petites suffusions sanguines comparables à celles que l'or observe dans les cas de piqûres d'insectes. L'irritation peut durer et persister quelquefois plusieurs jours après la piqûre.

Au point de vue de sa biologie, $B$. bursa est hématophage; on le trouve dans le plumage des volailles, très souvent à la base des plumes oil il voisine avec des Mallophages et d'autres Acariens plumicoles. Il est extrêmement rapide dans ses 
déplacements. Il quitte, en général, son hôte pendant la journée pour aller se réfugier dans les litières, le guano, les anfractuosités des murs de la volière, les toits, les nids des pondeuses. Cependant, comme nous avons pu le constater au laboratoire où nous l'avons mis en élevage dans des bacs à tiques, il n'est pas lucifuge, et on peut le trouver en abondance sur des volailles durant la journée, particulièrement sur des poules couveuses ou des poules malades se déplaçant difficilement. Cet acarien est un parasite intermittent qui quitte son hôte le matin au réveil pour revenir sur l'oiseau dès qu'il est de nouveau au repos sur son perchoir. $B$. bursa ne fuit pas la lumière, mais est plutôt sensible aux bruits et aux chocs.

Nous avons pu constater également que $B$. bursa ne quitte pas la poule sitôt la mor $L$ il se réfugie à l'extrémité des rémiges. On voit alors les parasites grouiller sur le côté opposé à celui sur lequel le cadavre est couché. Ce comportement vis-à-vis du cadavre de l'hôte a une grande importance au point de vue de la transmission possible des maladies aviaires, telles que choléra, spirochétose, etc. $\bar{A}$ ce sujet, des expériences sont en cours au Laboratoire Central de l'Élevage à Tananarive.

Si la femelle de $B$ : bursa est hématophage et a besoin de repas sanguins pour effectuer sa ponte, il ne semble pas' en' être de même du mâle, qui est très rare et que l'on trouve surtout dans les gîtes, refuges plus ou moins garnis de poussières et de guano. Les deux seuls'spécimens que nous ayons pu récolter ont été recueillis dans un panier en vannerie crasseux qui avait servi au transport de volailles parasitées et avait été abandonné dans des locaux malpropres. Ces deux mâles ne présentaient aucune trace de sang dans leur intestin. Il semble en être de même pour les protonymphes trouvées elles aussi dans le même biotope.

Etant donné la rareté des mâles, la reproduction aurait lieu, en leur absence, par parthénogénèse, comme on l'observe chez beaucoup d'espèces de Macronyssidae (Da Fonseca 1947).

Le rôle pathogène de $B$. bursa est très important et présente beaucoup d'analogie avec celui de Dermanyssus gallinae; d'une part, par les prélèvements répétés de sang comparables à de véritables saignées quand les parasites pullulent, d'autre part, par le prurit qu'il déclenche, obligeant l'oiseau à des grattages continuels qui nuisent à son bon entretien et à l'engraissement normal des volailles. Des poules couveuses fortement parasitées quittent souvent leur nid et abandonnent leur couvée à la suite d'attaques massives de $B$. bursa ; cela se voit couramment dans les petits élevages malgaches.

Il faut signaler, en outre; l'action toxique de la salive des Gamasidae qui n'est pas négligeable et qui, ajoutée à l'anémie, peut sans doute, dans certains cas d'infestation massive, déterminer la mort de I'hôte. Brumpt (1949) signale l'importance de l'action toxique de la salive chez Dermanyssus gallinae et prouve expérimentalement qu'elle intervient pour une large part dans le pouvoir pathogène de cet acarien.

Les caractères morphologiques des spécimens que rous avons dans notre collection sont identiques à ceux que donne Hirst en 1915 pour $B$. bursa, que ce soit pour la femelle, le mâle ou la protonymphe.

Nous tenons cependant à exposer brièvement la morphologie de ce parasite, tout au moins en ce qui concerne la femelle : longueur de l'idiosoma : 650 à $700 \mu$, corps ovale, écusson dorsal d'une seule pièce, en pointe vers l'arrière, chelicères en "tenaille», plaque sternale trapézoïdale comportant six soies, plaque génitale triangulaire, écusson anal piriforme, pilosité peu dense sur la partie du corps non recouverte par l'écusson dorsal. Pattes I et IV sensiblement égales et plus longues que les pattes II et $\Pi$.

La lutte contre ce parasite découle de sa biologie. Elle consiste essentiellement à le détruire dans les refuges qu'il occupe pendant le jour : rrurs, toits, sols, litières, guano, nids, etc. Tout d'abord un nettoyage complet des volières est nécessaire de manière à découvrir tous les interstices et fentes garnis de guano, poussières, etc., qui sont autant d'écrans protecteurs pour le parasite contre les divers produits acaricides que l'on emploiera pour le détruire. Il ne faut pas oublier, en outre, que cet acarien est extrêmement résistanl au jeûne el que l'abandon d'une volière pendant une année ne suffit pas à le faire disparaître.

Le nettoyage de la volière accompli, on peut utiliser, comme mode de destruction, les fumigations à base d'anhydride sulfureux en faisant brîler du soufre. C'est un excellent procédé extrêmement rapide, économique et efficace, mais malheureusement- présentant deux inconvénients majeurs :

1 - les risques d'incendie toujours possibles dans les poulaillers construits en matériaux inflammables;

$2^{\circ}$ la nécessité absolue d'avoir des locaux hermétiquement clos. Il faut; en effet, que les vapeurs de SO2 restent en. contact pendant plusieurs heures avec le parasite pour le tuer.

Un autre moyen consiste à utiliser des produits acaricides. Notons au passage le manque d'action total du. D.D.T., de même que celui des préparations à base d'arsenic utilisées pour la lutte contre les ixodes du gros bétail. L'arsenic, très toxique pour les acariens, n'est pas un acaricide de contact mais agit seulement par ingestion.

Dans le cas de $B$. bursa, seul un produit agissant par contact peut être efficace. 
L'isomère $\gamma$ de l'héxachlorocyclohexane, ou lindane, est le produit tout indiqué pour la destruction de ces acariens. On l'utilisera de préférence (H.C.H. mouillable) sous forme de bouillies répandues par pulvérisations appliquées sur les murs, sols, toits, nids, etc. Pour le déparasitage des volailles, on pourra utiliser "l'exterminateur Lagrange », préconisé par Neveu-Lemaire en 1912, sorte de boite dans laquelle on met l'oiseau et où l'on fait brûler du soufre. On peut également appliquer des poudres à base de H.C.H., d'un emploi beaucoup plus facile et moins dangereux.

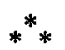

Au cours des expériences et observations faites au Laboratoire Central de l'Élevage à Tananarive sur le parasitisme des poules à Madagascar par $B$. bursa, nous avons été surpris de trouver un Tyroglyphus (Latreille, 1796), en nombre très important sur des poules malades provenant d'un élevage européen de la région de Tananarive.

Les Tyroglyphes qui sont des sarcoptidés détritiphages à respiration cutanée ont des biotopes extrêmement variés ; c'est ainsi que Tyroglyphus farinae (De Geer, 1808), parasite la farine, le bié et le pain. Dans les greniers, il peut se multiplier à l'extrême, au point qu'on le ramasse à la pelle (Guiart, 1922). Tyroglyphus siro (Linné, 1758), est particulièrement abondant sur la croûte et dans les yeux des fromages tels que le parmesan, le chester. le gruyère et le hollande. Tyroglyphus entomophagus (Laboulbène, 1852), attaque les collections d'insectes. Tyroglyphus longior (Gervais, 1844). parasite les vanilles entreposées, et Lecomte, dans son travail sur le Vanillier en 190I, dit : « les gousses sont fréquemment attaquées aussi dans les magasins par un acarien (Tyroglyphus spp.) qui ronge la surface du fruit ».

Chalot et Bernard, en 1918, signalent que la vanille entreposée dans les magasins, en France ou aux Colonies, est très souvent parasitée par un acarien blanchâtre (Tyroglyphus spp.).

Bouriquet, en 1941, signale l'importance à Madagascar d'un Tyroglyphus spp. qui parasite les gousses de vanille, occasionnant de gros dégâts dans les magasins. La détermination exacte du parasite dont parle l'auteur ne semble pas avoir été faite; les dessins qui en sont donnés et le manque de description précise ne permettent pas de savoir s'il s'agit de $T$. siro ou d'une autre espèce.

D'après le même auteur, la vanille en paquets est particulièrement parasitée par les «mites ». La cause favorisant l'apparition du mitage serait une fermentation des gousses au niveau des blessures faites aux fruits au moment du ramassage. Le Tyro- glyphe serait associé à la présence d'une levure qui apparaitrait au cours de fermentations, comme c'est fréquemment le cas pour certains acariens trouvés dans des vins mal soignés (Trouessart, 1897). Le Tyroglyphe n'apparaitrait qu'après le champignon et, dans le cas de la vanille. l'association acarien-levure serait constante.

Au point de vue du rôle pathogène des Tyroglyphes chez l'homme, ces acariens sont trouvés souvent dans le tractus intestinal, mais plus fréquemment à la surface de la peau où ils déterminent des lésions cutanées plus ou moins prurigineuses, d'où les noms de "gale vanillique », "copraitch 》 donnés à ces diverses affections. Beaucoup plus rarement, ces acariens ont été signalés dans des tumeurs cutanées, des kystes parasitaires et dans les voies urinaires.

C'est ainsi que Gedoelst, en 1911, dans son ouvrage de parasitologie, signale que $T$. farinae et $T$. siro ingérés par l'homme avec du pain et du fromage peuvent provoquer du catarrhe intestinal. D'après Smart (1948), les Tyroglyphes, en plus des accidents cutanés et intestinaux qu'il sont susceptibles de provoquer chez l'homme, peuvent faire apparaitre des troubles d'allure asthmatiforme. T. longior var. castellanii (Hirst; 1912), pourrait vivre pendant très longtemps dans le tractus intestinal de l'homme et même s'y reproduire. C'est quand l'infestation devient particulièrement massive qu'apparaissent les symptômes rappellant l'asthme.

La gale vanillique ou vanillisme, caractérisée par une éruption prurigineuse de la face, du cou et des mains, s'accompagne souvent de conjonctivite et de coryza. On l'observe sur des ouvriers qui manipulent des vanilles moisies et mitées dans les entrepôts ou les distilleries (Gedoelst, 1911 ; Guiart, 1922 ; Brumpt, 1948 ; Smart, 1948; Matheson. 1950). T. farinae peut, quand il parasite massivement la farine ou le blé, provoquer des éruptions cutanées chez les ouvriers qui manient ces produits, tels que les meuniers ou les boulangers (Gedoelst, 1911 ; Neveu-Lemaire, 1942 ; Smart, 1948).

Le "copra-itch", observé chez l'homme aux Indes (Ceylan), est une dermatite prurigineuse causée par Tyroglyphus longior var. castellanii que l'on trouve dans le coprah et à la surface des noix de coco (Hirst, 1912).

Comme autres localisations, T. longior a été vu dans le pus de lésions cutanées (Brumpt, 1927). Le même auteur relate un cas où Histiogaster spermaticus (Trouessart, 1902), a été trouvé aux Indes par le Docteur Pye Smith dans le liquide extrait par ponction d'un kyste du pli de l'aine et adhérent au sommet du testicule droit. Le kyste renfermait, outre $60 \mathrm{~g}$ d'un liquide clair limpide, un nombre considérable d'acariens vivants à tous les stades 
de leur évolution. Il est probable, fait remarquer Brumpt, que ce Tyroglyphidae détriticole avait été introduit par une sonde malpropre au cours d'un sondage récent fait pendant un accès de paludisme pernicieux.

L'infection du tractus urinaire chez l'homme par les Tyroglyphes est assez rare; cependant Meckie, en 1926, relate un cas d'infection vésicale par Tarsonemus floricolus C. et F., Glyciphagus domesticus De Geer et Tyroglyphus longior. Lucasse et Borghers, en 1953, décrivent 5 cas d'acaridiase urinaire chez l'homme par des Tyroglyphes. Dans un cas, ils supposent qu'il s'agit de $T$. longior. La déterminaison exacte du parasite en cause ne semble pas avoir été faite. Les symptômes qui prédominaient étaient surtout constitués par de violentes douleurs au niveau de l'hypocondre et par de l'hématurie.

En médecine vétérinaire, Neveu-Lemaire (1942) fait remarquer que si $T$. farinae vit ordinairement dans les issues, la farine et le blé, il peut, quand. il devient trop nombreux dans ces produits, envahir le plumage des volailles et causer d'impórtants dégâts dans les élevages avicoles. A notre connaissance, c'est le seul auteur qui ait parlé du parasitisme des volailles par les Tyroglyphes.

Les Tyroglyphidae sont de très petits acariens aveugles et dépourvus de stigmates. A la différence des Sarcoptinae, agents des gales humaines et animales, ils ont sensiblement leurs quatres paires de pattes semblables.

Description du parasite :

Mâle : couleur jaune sale, longueur $380 \mu$, largeur $160 \mu$.

Face dorsale:

Gnathosoma de forme sensiblement triangulaire; longueur $68 \mu$, largeur à la base $47 \mu$. Il est légèrement rétréci à sa partie moyenne.

Propodosoma : longueur $80 \mu$, contre $136 \mu$ à sa plus grande largeur. Sa partie moyenne présente deux soies de longueur moyenne disposées côte à côte. De part et d'autre de ces deux dernières sont placées deux soies courtes dressées, trapues et fortement palmées. Une rangée de quatre longues soies dans la partie postérieure de cette région est parallèle au sillon séjugal très nettement marqué et légèrement infléchi vers l'arrière dans sa partie moyenne.

Hystérosoma : longueur $242 \mu$, largeur $160 \mu$.

Il présente dans sa partie moyenne et antérieure deux paires de longues soies dont l'une est latérale et l'autre sublaterale; dorsalement et juste en arrière du sillon séjugal, deux soies courtes. Plus en arrière, au niveau des pattes III, existent deux paires dé soies moyennes médiodorsales; deux courtes soies sont placées plus en arrière et de part et d'autre de ces dernières. Deux très longues soies marquent la partie antérieure de l'opisthosoma. La partie postérieure de cette dernière région est occupée par trois paires de longues soies dont la plus antérieure est en position sublatérale. Enfin, il existe deux paires de longues soies terminales.

Dorsalement, l'hystérosoma présente ses bords latéraux sensiblement parallèles alors que son extrémité postérieure est régulièrement arrondie. Au niveau du sillon séjugal, le corps de l'acarien est marqué d'un très fort rétrécissement.

Face ventrale :

Gnathosoma : chélicères très puissantes avec extrémités en pince très forte. Face interne des mors pourvue de dents très fortes. Un des mors est mobile alors que l'autre est fixe. Les deux mors sont sensiblement égaux en longueur et en puissance.

Podosoma : présence d'une soie courte et raide sur les coxae I, III et IV. Le métapodosoma supporte, juste en arrière du sillon séjugal et éntre les coxae III et IV, une paire de soies courtes.

L'organe génital est situé entre les coxae IV. Il existe deux paires de ventouses génitales de chaque côté de l'organe copulateur. Pénis rectiligne. Entre chaque paire de ventouses, on remarque une très fine et très courte soie raide.

L'uropore est en forme de fente longitudinale; il est flanqué postérolatéralement de deux ventouses anales présentant une échancrure dans leur bord antérieur, ainsi qu'une très petite soie à leur coin antéro-externe.

Postérieurement et en position subterminale existent quatre longues soies.

Pattes : elles sont toutes sensiblemént de la même longueur et sont plutôt élancées que trapues. Elles comportent les six articles classiques : coxa, trochanter, fćmur, génual, tibia, tarsc.

Les trochanters des pattes II et III sont pourvus d'une petite soie. Tous les tibias présentent à leur extrémité apicale une longue soie raide de longueur égale ou sensiblement plus longue que le tarse.

Il existe une griffe à l'extrémité de chácun des tarses dont les I et II présentent dans leur partie moyenne un appendice court, trapu, cylindrique et à l'extrémité obtuse.

Femelle : couleur identique à celle du màle, longueur $520 \mu$, largeur $260 \mu$.

Face dorsale :

Gnathosoma : longueur $68 \mu$, largeur $60 \mu_{i}$ sensiblement triangulaire.

L'idiosoma présente une chaetotaxie identique à celle du mâle au point de vue disposition et longueur des différentes soies. L'allure générale de cette face est plus trapue et moins élancée que celle du mâle. 
Face ventrale:

Antérieurement, même disposition que chez le mâle, au point de vue implantation des soies.

Existence de ventouses génitales.

Complexe génital en forme de $\mathrm{V}$ renversé.

Uropore longitudinal avec quatre paires de soies disposées de part et d'autre et d'avant en arrière. La paire terminale est de moyenne longueur alors que les trois autres sont courtes.

Quant aux soies terminales, la femelle présente deux paires de longues soies qui n'existent pas chez le mâle. Une est placée juste en arrière de l'uropore, l'autre est externe.

\section{Pattes :}

Chez la femelle, les pattes I et II sont plus trapues et plus épaisses que les III et IV.

Remarques : Cet acarien présente tous les caractères du genre Tyroglyphus (Latreille, 1796). Il est à rapprocher de $T$. longior (Gervais, 1844) et de T. longior var. castellanii (Hirst, 1912) dont il possède la même chaetotaxie dorsale, sauf cependant un détail supplémentaire, la présence d'une courte soie palmée sur la partie antérieure du propodosoma. - Sa face ventrale présente des différences notoires avec T. longior.

Absence de soie sur le trochanter IV, épimères I plus minces, présence d'une paire de soies sur le métapodosoma et entre les coxae III et IV.

La détermination exacte de l'espèce est en cours.

Au point de vue du pouvoir pathogène de cet acarien sur les volailles, nous avons pu constater au laboratoire que ce parasite se tient en permanence dans le plumage et provocue, comme les Mallophages et $B$. bursa, des démangeaisons incessantes quand il est en grande abondance. Contrairement à $B$. bursa, il quitte son hôte sitôt après la mort de ce dernier pour se réfugier dans des débris de toutes sortes. Cet acarien est occasionnellement hématophage : à l'examen microscopique, nous avons trouvé du sang dans l'intestin de certains spécimens.

Le biotope normal de ce tyroglyphe reste à trouver car cet acarien doit avoir des mours détriticoles.

La destruction de ce parasite peut se faire, comme pour $B$. bursa, par épandage de produits à basc d'H.C.H.

Quant à la prophylaxie, elle nécessite la connaissance des lieux de reproduction et des réservoirs naturels, farines, tourteaux, etc. de ce Tyroglyphus sp. (1).

\section{Laboratoire Central de l'Élevage et des Industries Animales, Tananarive.}

(1) Nous tenons à remercier particulièrement M. le Professeur J. Colas-Belcour, de l'Institut Pasteurde Paris, qui nous a aidé dans nos déterminations, et l'Institut de la Recherche Scientifique de Madagascar qui nous a ouvert les portes de sa bibliothèque et nous a ainsi permis de faire la majeure partie de la bibliographie de cet article.

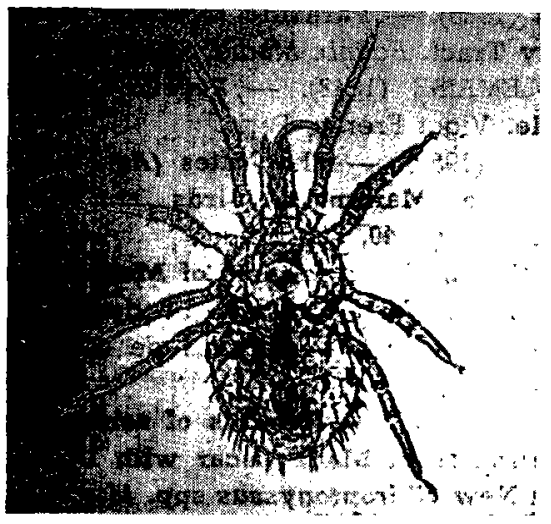

I. - Bdellonyssus bursa femelle.

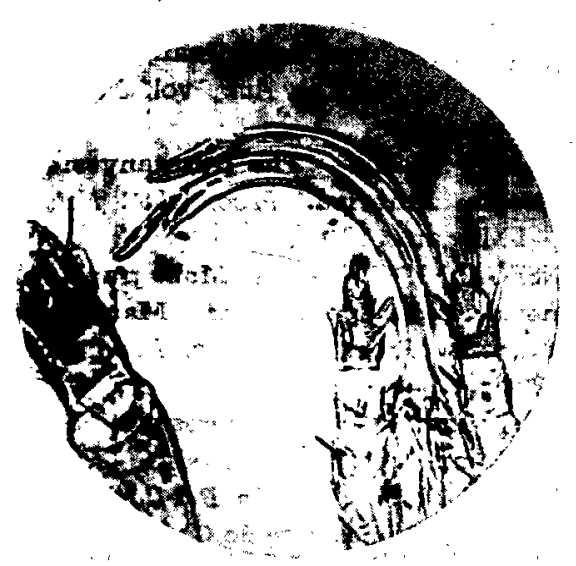

II. - Chélicères de B. bursa (extrémité libre). 


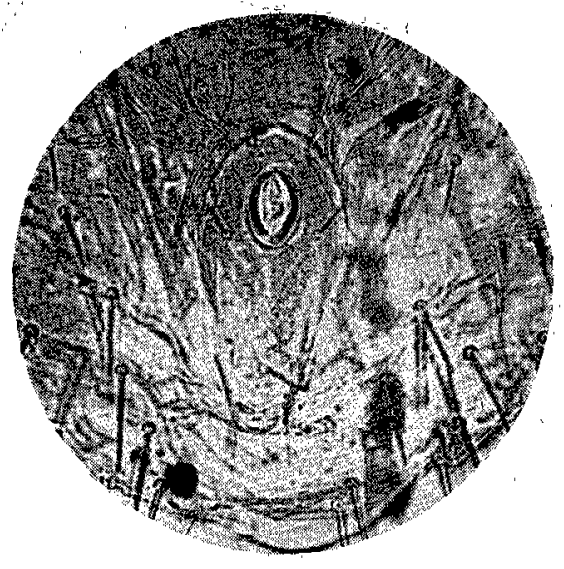

III. - Écusson sternal de B. bursa.

\section{BIBLIOGRAPHIE}

ANDRÉ (M.) (1949). - Cours sur les Acariens. Office de la Recherche Scientifique d'outre-mer, Paris,

BOURIQUET (G.) (1941). - Contribution à l'étude des altérations de la vanille préparée (Moisissures et mites). Bull. Acad. Malgache, t. XYIV, 65-81.

BERLESF. (A.) (1888). '- Bull. Soc. Ent. Ital. t. XX, 171.

BERLESE (A.) (1918). - Centuria quarta di Acari nuovi. Resdia, 13 115-190.

BRIJMPT (E.) (1927). - Précis de Parasitologie. Masson Paris.

BRUMPT (E.) (1949). - Précis de Parasitologie. Masson, Paris.

CHALOT et BERNARD (U.) (1920). - Culture et préparation de la vanille. Larose, Paris.

EWING (H.-E.) (1911). - The English Sparrow as an Agent in the Dissemination of Chicken and Bird Mites. The $A u k$, vol. XXVIII nouv. sér. $335-340$.

EWING (H.-E.) (1923). - The Dermanyssid Mites of North America. Proc. U.S. Nat. MuS., vol. LXII, art. 13,1.

Da FONSECA (1947). - A Monograph of the Genera and Species of Maeronyssidae Oudemans, Proc. Zool. Soc. Lond., part. II, 249-334.

- GEDOELST (L.) (1911). - Synopsis de Parasitologie. Henri Lemartin, Bruxelles, 173.

GRETILLAT (S.) (1955). - Un Bdellonyssus Fonseca (1941) ectoparasite de Gallus domesticus Linn. à Madagascar. Rapport annuel 1955. Laboratoire Central de l'Elevage à Tananarive.

GUIART (J.) (1922). - Précis de Parasitologie. Baillière et fils, Paris.

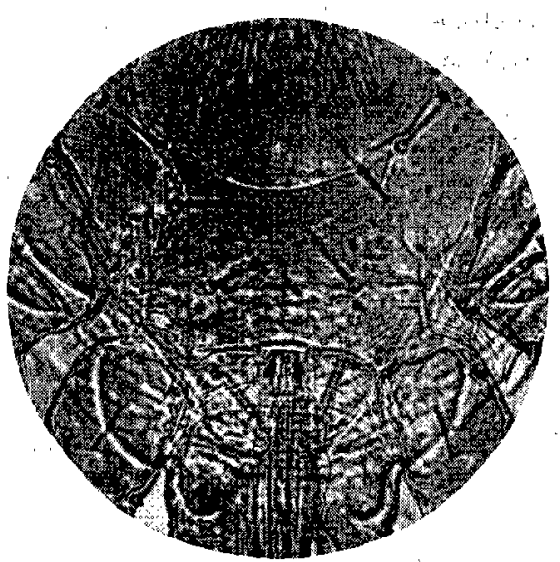

IV. - Écusson anal de B. bursá.

HIRST (S.) (1912). - On two New Parasitic Acari of the Genus Leiognathus. Bull. Ent. Res., 3, 369-372.

HIRST (S.) (1912). - Report on the Mites causing

Copra Itch. Journ. of Tropic. Medicine, 374.

HIRST (S.) (1915) - On a Widely distributed Gamasid Mite. (Meiognathus morsitans). Parasitic on the Domestic Fowl. Bull. Ent. Res., 55-58.

HIRST (S.) (I921). - On some New Parasitic Mites. Proc. Zool. Soc. Lond., 769.

HIRST (S.) (1922). -- Mites Injurious to Domestic Animals. Brit. Mus. Nat. Hist. Econ. Ser., 13, 82.

LECOMTE (H.) (1901), - Le Vanillier. Naud, Paris. LUCASSE (C.-H.-R.) et BORGHERS (G.) (1953). Acaridiase humaine du tractus urinaire. Ann. Soc. Belge Méd. Trop., 5, vol. 33, 451-456.

MÁTHESON (1950) ... - Medical Entomology. 2th edit. Comstock Publishing Cy, New-York.

MECKIE (1926). - Parasitic Infection of the Urinary Tract. Edimb. Medic. 33, 708-719.

NEVEU-LEMAIRE (1942). - Précis de Parasitologie. Vigot Frères, Paris.

RADFORD (1950), - The Mites (Acarina) Parasitic on Mammals, Birds and Reptiles. Parasitology, 40, 566-594.

SMART (J.) (1948). - Insects of Medical Importance. Brit. Mus. Nat. Hist. London.

TROUESSART (1897). - Annales de Gastronomie, 554.

ZUMPT (F.) (1950). - Records of some Parasitic Acarina from Madagascar with Description of a New Chiroptonyssus spp. Mem. Inst. Sci. Madagas., vol. IV, fasc. I, 165-173.

ZUMPT (F.) and P.ATTERSON (1951). - Ethiopian Laelaptid Mites. J. Ent. Soc. S. Afr., 2, vol. XIV, 63-93. 


\section{SUMMARY}

Two acaridan parasites of Gallus domesticus Lin. in Madagascar

(Bdellonyssus bursa Berlese 1888 and Tyroglyphus sp.)

The author wishes to draw attention to the existence of Bdellonyssus bursa Berlese 1888, as an extremely common parasite amongst poultry in Madagascar. He points out that important damage can be caused by this parasite in poultry farming.

Under certain conditions not yөt quite clear, a Tyroglyphydae, Tyroglyphus sp., may infest poultry.

The methods of control are particularly of a prophylactic nature : periodical cleaning out of fowlhouses, spraying of acaricide products composed of B.H.C. on the ground, the walls and the roofs of fowl-houses.

\section{RESUMEN}

Dos ácaros parásitos de Gallus domesticus Lin. en Madagascar (Bdellonyssus bursa Berlese 1888 y Tyroglyphus sp.)

El autor señala la existencia de Bdellonyssus bursa, 1888, en Madagascar, como un parásito muy frecuente en las aves. Llama la atención sobre los estragos que este parásito puede producir en los ganaderias. las gallinas.

En ciertas condiciones, aun no dilucidadas, un Tyroglyphydae. Tyroglyphus sp., puede parasitar

La conducta a seguir para la lucha contra estos ectoparasitos es sobre todo de orden profiláctico : limpieza periódica de los viveros, pulverización de productos acaricidas a base de H.C.H. sobre las paredes, suelos y techos de los gallineros. 